\title{
ESOFAGITE EOSINOFÍLICA EM 29 PACIENTES PEDIÁTRICOS
}

\author{
Cristina Targa FERREIRA', ${ }^{1}$, Mário C. VIEIRA ${ }^{3}$, Sandra Maria Gonçalves VIEIRA², \\ Giovana Stival da SILVA ${ }^{3}$, Danielle Reis YAMAMOTO ${ }^{3}$ Themis Reverbel da SILVEIRA²
}

\begin{abstract}
RESUMO - Racional - A esofagite eosinofílica é uma entidade recentemente descrita, caracterizada por sintomas esofágicos, semelhantes aos da doença por refluxo gastroesofágico e importante eosinofilia esofágica. Objetivo - Apresentação de 29 pacientes com esofagite eosinofílica, discutindo as características clínicas, diagnóstico, tratamento e evolução. Métodos - Foram identificados 29 pacientes (22 do sexo masculino) com idade entre 1 e 18 anos, nos quais as biopsias de esôfago demonstraram contagem de 20 ou mais eosinófilos/campo de grande aumento, sem infiltração eosinofílica em antro e/ou duodeno. Avaliaram-se as manifestações clínicas, achados endoscópicos e histológicos, tratamento e evolução. Resultados - Os sintomas mais freqüentes foram vômitos em 15 pacientes (52\%) e dor abdominal em 11 (38\%). Os pacientes com idade inferior a 4 anos apresentavam recusa alimentar e baixo peso. Os com idades entre 5 e 8 anos apresentavam predominantemente dor abdominal e/ou pirose e/ou vômitos. Os pacientes com mais de 8 anos apresentavam dor abdominal, disfagia e/ou impactação alimentar eventual. Os achados endoscópicos incluíram estrias verticais em 14 pacientes (48\%), pontilhado branco em 12 (41\%), anéis circulares em 2 (7\%) e esofagite erosiva em 3 (10\%). Em sete pacientes a endoscopia foi normal (24\%). O tratamento incluiu fluticasona tópica em 19 pacientes e restrição dietética em 7. Os pacientes acompanhados apresentaram resposta favorável ao tratamento, com melhora ou remissão dos sintomas. Onze pacientes que foram submetidos a endoscopia de controle pós-tratamento apresentaram diminuição significativa do número de eosinófilos no esôfago. Conclusões - A esofagite eosinofílica deve ser considerada quando há sintomas de refluxo, que não respondem ao tratamento habitual. Os exames endoscópicos devem ser acompanhados de biopsias com análise detalhada do número de eosinófilos.
\end{abstract}

DESCRITORES - Esofagite. Eosinofilia. Criança.

\section{INTRODUÇÃO}

Na última década, uma entidade clínico-patológica distinta, denominada esofagite eosinofílica (EE), provavelmente imunomediada, tem sido progressivamente descrita em crianças e em adultos ${ }^{(2)}$. Embora o primeiro caso de EE tenha sido publicado em $1978^{(23)}$, as características clínicas e histopatológicas dessa enfermidade só passaram a ser definidas na década de $90^{(5,25,34)}$.

A história natural ainda não está bem definida, mas os estudos de mais longo prazo têm mostrado um curso persistente ou recurrente em mais de $90 \%$ dos casos de $\mathrm{EE}^{(2,4,11,25)}$.

O tratamento clínico tem sido empírico, incluindo o uso de corticóides tópicos, antagonistas de receptores dos leucotrienos, dietas de eliminação (fórmulas de aminoácidos) ou dietas de restrição (exclusão de grupos de alimentos da dieta $)^{(2,12,20,26)}$.

As observações clínicas têm sugerido fortemente que essa é mais uma doença alérgica, com vários pontos a serem esclarecidos e vários desafios a serem vencidos.

Além do aumento da freqüência das alergias, mais do que dois terços dos artigos sobre EE foram publicados nos últimos 3 anos. Sendo assim, é muito importante que os pediatras estejam atualizados em relação à ela ${ }^{(12,26)}$.

O objetivo deste estudo foi apresentar 29 crianças e adolescentes com EE, provavelmente a primeira série de casos pediátricos publicada no Brasil, discutindo e revisando as características clínicas, o diagnóstico e a possível evolução dessa entidade.

\section{MÉTODOS}

Trata-se de estudo observacional e descritivo que envolve pacientes pediátricos, com diagnóstico de EE, atendidos em dois serviços de gastroenterologia pediátrica das cidades de Porto Alegre, RS, e Curitiba, PR, ambas localizadas na região sul do Brasil .

Os pacientes foram submetidos a endoscopia digestiva alta para investigação de sintomas relacionados ao tubo digestivo superior, realizada sob anestesia geral ou sedação profunda, com endoscópios flexíveis. Foram obtidas biopsias de esôfago, antro e duodeno em todos os pacientes, já que é rotina em endoscopia pediátrica, com fórceps de biopsias convencionais.

IInstituto do Aparelho Digestivo, Porto Alegre, RS; ${ }^{2}$ Hospital de Clínicas de Porto Alegre, RS; ${ }^{3}$ Hospital Pequeno Príncipe, Curitiba, PR.

Corrrespondência: Dra. Cristina Targa Ferreira - Rua Pedro Weingartner, 105/202 - 90430-140 - Porto Alegre, RS. E-mail: cristinatarga@terra.com.br 
O diagnóstico de $\mathrm{EE}$ foi obtido através do estudo histológico das biopsias do esôfago distal, que foram revisadas por um único patologista, em cada cidade, e foram consideradas diagnósticas quando apresentavam mais de 20 eosinófilos por campo de grande aumento, de acordo com o preconizado pela literatura ${ }^{(2,12,28,31,32)}$. O número de eosinófilos foi obtido utilizando-se o critério de contagem de seu número, superior a 20 por campo de grande aumento, na área de maior concentração dos mesmos. Portanto, os pacientes foram recrutados após o resultado das biopsias com contagem de eosinófilos.

A coloração usada foi hematoxilina-eosina em todas as biopsias. O diagnóstico foi sempre histológico e realizado quando não havia infiltração eosinofílica concomitante em antro e/ou duodeno.
Foram avaliados os sintomas de apresentação dos pacientes, os resultados das endoscopias e das biopsias, o tratamento e a evolução, quando disponíveis.

\section{RESULTADOS}

Foram estudados 29 pacientes, 11 provenientes de Porto Alegre e $18(62 \%)$ de Curitiba, em um estudo colaborativo desses dois centros (Tabela 1).

As idades variaram de 1 a 18 anos, com mediana de 7 anos. Vinte e dois pacientes eram do sexo masculino (76\%).

Os sintomas mais freqüentes foram vômitos em 15 pacientes (52\%) e dor abdominal em 11 (38\%). Todos os pacientes com 4 anos ou menos (nove) apresentavam recusa alimentar e baixo

TABELA 1. Achados clínicos dos 29 pacientes portadores de EE

\begin{tabular}{|c|c|c|c|c|c|}
\hline N/Sexo & Idade & Queixa & Endoscopia & $\begin{array}{l}\text { Eosinófilos/ } \\
\text { campo } \\
\text { pré/pós tratamento }\end{array}$ & Tratamento / evolução \\
\hline $1 / \mathrm{F}$ & 10 & Vômitos noturnos há 3 anos/dor epigástrica & Estrias verticais & 20 & Montelukast + dieta/melhora clínica \\
\hline $2 / \mathrm{F}$ & 1 & Recusa alimentar/irritabilidade & Normal & $20 / 0$ & $\mathrm{PF}+\mathrm{IBP} /$ melhora clínica/ endo \\
\hline $3 / \mathrm{M}$ & 4 & $\begin{array}{l}\text { Disfagia + desnutrição } \\
\text { (paralisia cerebral) }\end{array}$ & $\begin{array}{l}\text { Estrias verticais/ pontilhado branco/ } \\
\text { estenose }\end{array}$ & $20 / 12$ & $\begin{array}{l}\text { Dilatação+dieta AA p/SNG/melhora clínica/ } \\
\text { endo/retorno dos sintomas e eosinofilia } \\
(50 \mathrm{p} / \mathrm{c}) \text { após dieta polimérica }\end{array}$ \\
\hline $4 / \mathrm{M}$ & 8 & Dor epigástrica/vômitos & $\begin{array}{l}\text { Estrias verticais/ } \\
\text { pontilhado branco }\end{array}$ & $50 / 20$ & $\begin{array}{l}\mathrm{PF} / \mathrm{melhora} \text { clínica/ endoscopia ficou com } \\
20 \text { Eosinófilo/campo }\end{array}$ \\
\hline $5 / \mathrm{M}$ & 14 & Dor abdominal & Estrias verticais/ pontilhado branco & 25 & Dieta hipoalergênica/ melhora clínica \\
\hline $6 / \mathrm{M}$ & 10 & Disfagia/dor retroesternal & Estrias verticais/ esofagite erosiva & $20 / 2$ & $\mathrm{PF}+\mathrm{IBP} /$ melhora clínica/ endo \\
\hline $7 / \mathrm{M}$ & 1 & Vômitos/irritabilidade/baixo peso & Estrias verticais/ pontilhado branco & $50 / 1$ & $\begin{array}{l}\mathrm{PF}+\text { dieta sem } \mathrm{LV} / \text { ovo/soja/ frutos do mar } \\
\text { /melhora clínica/endo }\end{array}$ \\
\hline $8 / \mathrm{M}$ & 13 & Disfagia há vários anos & Normal & $50 / 2$ & Montelukast-melhora clínica/endo \\
\hline $9 / \mathrm{M}$ & 5 & Pirose há 2 meses, baixo peso & Estrias verticais & 40 & $\mathrm{PF}$ \\
\hline $10 / \mathrm{M}$ & 5 & Dor epigástrica há 4 meses & Normal & 25 & ?? outro serviço \\
\hline $11 / \mathrm{M}$ & 6 & Vômitos e dor epigástrica & Esofagite erosiva & 25 & $\mathrm{PF}$ \\
\hline $12 / \mathrm{M}$ & 1 & Vômitos há >1 ano & Normal & 20 & ?? outro serviço \\
\hline $13 / \mathrm{F}$ & 6 & Anemia/vômitos/dor epigástrica & Normal & 30 & ?? outro serviço \\
\hline $14 / \mathrm{M}$ & 1 & Vômitos/perda de peso & Estrias verticais & $20 / 0$ & PF-melhora clínica/endo \\
\hline $15 / \mathrm{M}$ & 7 & Baixo peso/vômitos & Estrias verticais/ pontilhado branco & $>60$ & $\mathrm{PF}+\mathrm{IBP} /$ melhora clínica \\
\hline $16 / \mathrm{M}$ & 18 & $\begin{array}{l}\text { Baixo peso/vômitos/impactação/ } \\
\text { disfagia/irmão com EE (15) }\end{array}$ & Estrias verticais/ pontilhado branco & $>60$ & $\mathrm{PF}+\mathrm{IBP} /$ melhora clínica \\
\hline $17 / \mathrm{M}$ & 8 & Disfagia/impactação/baixo peso & Estenose/pontilhado branco & $40 / 1$ & Dilatação+PF+IBP/melhora clínica/endo \\
\hline $18 / \mathrm{M}$ & 11 & Impactação alimentar eventual & Pontilhado branco & $30 / 2$ & $\mathrm{PF}+\mathrm{IBP}+$ dieta/melhora clínica/endo \\
\hline $19 / \mathrm{F}$ & 17 & Vômitos/disfagia/impactação & $\begin{array}{l}\text { Acalásia/restos alimentares/ } \\
\text { pontilhado branco }\end{array}$ & $>50 / 1$ & $\begin{array}{l}\text { Dilatação+PF+dieta sem LV e derivados/ } \\
\text { carne vermelha } \\
\text { melhora clínica/endo }\end{array}$ \\
\hline $20 / \mathrm{M}$ & 4 & Recusa alimentar/baixo peso & $\begin{array}{l}\text { Estrias verticais/ } \\
\text { erosão }\end{array}$ & 20 & $\mathrm{PF}+\mathrm{IBP} /$ melhora clínica \\
\hline $21 / \mathrm{M}$ & 6 & Baixo peso/vômitos & Normal & $30 / 2$ & $\mathrm{PF}+\mathrm{IBP} /$ melhora clínica/ endo \\
\hline $22 / \mathrm{M}$ & 12 & Disfagia/impactação eventual/vômitos & Pontilhado branco & 30 & $\mathrm{PF}+\mathrm{IBP} /$ melhora clínica \\
\hline $23 / \mathrm{F}$ & 8 & Dor abdominal/impactação eventual & Pontilhado branco & 30 & $\mathrm{PF}+\mathrm{IBP} /$ melhora clínica \\
\hline $24 / \mathrm{F}$ & 9 & Dor epigástrica/impactação eventual & Anéis circulares & $>30$ & $\mathrm{PF}+\mathrm{IBP} /$ melhora clínica \\
\hline $25 / \mathrm{M}$ & 1 & Vômitos/baixo peso/recusa alimentar & Normal & 20 & PF \\
\hline $26 / \mathrm{M}$ & 2 & Vômitos/dor/baixo peso/recusa alimentar & Pontilhado branco & 30 & $\mathrm{PF}+\mathrm{IBP}+$ dieta sem $\mathrm{LV}$ e derivados \\
\hline $27 / F$ & 2 & Dor abdominal & Estrias verticais & 22 & ?? outro serviço \\
\hline $28 / \mathrm{M}$ & 10 & Disfagia/engasgos & Estrias verticais & 25 & PF-melhora clínica \\
\hline $29 / \mathrm{M}$ & 10 & $\begin{array}{l}\text { Disfagia/vômitos/gastrostomia/ } \\
\text { (paralisia cerebral) }\end{array}$ & Estrias verticais e anéis circulares & 30 & Dieta com AA-melhora clínica \\
\hline
\end{tabular}


peso. Os pacientes com mais de 8 anos apresentavam dor abdominal e/ou disfagia e/ou impactação alimentar eventual (11). Aqueles com idades entre 5 e 8 anos queixavam-se predominantemente de dor abdominal e/ou pirose e/ou vômitos eventuais (nove), sendo que quatro deles apresentavam baixo peso (Tabela 1).

Um paciente (de 8 anos) apresentou impactação alimentar muito sintomática e teve que ser submetido a endoscopia de urgência para retirada de restos alimentares e dilatação esofágica. Uma outra paciente de 17 anos, apresentou exame radiológico sugestivo de acalásia da cárdia e episódios importantes de impactação alimentar.

Um adolescente de 18 anos foi investigado e submetido a endoscopia com biopsias após o diagnóstico do irmão, de 7 anos, pois apresentava sintomas semelhantes, não investigados.

Os achados endoscópicos do esôfago incluíram estrias verticais em 14 pacientes (48\%), pontilhado branco em 12 (41\%), anéis circulares concêntricos em $2(7 \%)$ e 3 pacientes $(10 \%)$ apresentavam erosão linear no esôfago terminal. Sete pacientes não apresentavam anormalidades ao exame endoscópico (24\%).

Dois pacientes (4 e 8 anos) apresentavam estenose de esôfago no momento do diagnóstico e necessitaram submeterse a dilatação esofágica. A paciente de 17 anos foi submetida a várias endoscopias por estar obstruída e com restos alimentares no esôfago, necessitando dilatações esofágicas.

Todos os pacientes apresentavam média de 20 ou mais eosinófilos por campo de grande aumento (média de 32/campo), com números que variavam de 20 a mais de 60/campo, no momento do diagnóstico.

Dezenove pacientes acompanhados, receberam tratamento inicial com fluticasona tópica, que era administrada em dois jatos pela manhã e dois à noite, na boca, com deglutição posterior e manutenção de jejum alimentar por, pelo menos 1 hora de jejum após. Todos apresentaram boa resposta clínica imediata ao início do tratamento. Doze pacientes receberam também inibidores de bomba de prótons (IBP), omeprazol na maioria dos casos, previamente ou concomitante à fluticasona.

Sete pacientes receberam indicação de restrição dietética, sem leite de vaca e/ou derivados e carne vermelha, durante o tratamento. Um paciente, de 4 anos, com dano neurológico, ficou 4 meses sob dieta com fórmula de aminoácidos por sonda nasogástrica, com boa resposta clínica e histológica ao tratamento. Este mesmo paciente teve recidiva dos sintomas e das alterações endoscópico-histológicas, quando do retorno da dieta polimérica. Outro paciente, com dano neurológico e gastrostomia, recebeu dieta com hidrolisado protéico, mas ainda não teve acompanhamento clínico a longo prazo.

Onze pacientes foram submetidos a endoscopia de controle pós-tratamento, com biopsias de esôfago. Todos apresentaram diminuição significativa do número de eosinófilos por campo, sempre para menos do que 20 eosinófilos/campo (média de 4 eosinófilos/campo de grande aumento), com exceção de um paciente de 8 anos, que permaneceu com 20 eosinófilos/campo na biopsia de controle.

Cinco dos pacientes desta série apresentam mais de 1 ano de evolução: o paciente de 8 anos, com estenose, não apresentou recidiva da impactação alimentar e da disfagia, durante 2 anos de acompanhamento. Os demais apresentam episódios eventuais de dor abdominal. Esses pacientes apresentam alergia respiratória, com episódios recurrentes de rinites e broncoespasmo. Os outros pacientes ainda têm tempo curto de acompanhamento para que se possa especificar a evolução em prazo mais longo.

Os pacientes em acompanhamento por tempo mais longo, tiveram boa resposta ao tratamento inicial: ganho de peso ponderal, melhora do apetite, ausência de sintomas de dor abdominal e desaparecimento da disfagia e impactação alimentar. A paciente de 17 anos apresentou vários episódios de disfagia e impactação, sendo submetida a endoscopia pelo menos 2 vezes por ano.

\section{DISCUSSÃO}

Este estudo abrange 29 casos de EE em crianças e adolescentes, provavelmente a primeira série de pacientes pediátricos a ser descrita na literatura brasileira, demonstrando a existência dessa enfermidade também em países em desenvolvimento. FURTADO e HERCULANO Jr. ${ }^{(13)}$ já descreveram 10 casos em pacientes com idades entre 16 e 55 anos, em Fortaleza, CE.

A maioria dos pacientes desta casuística era do sexo masculino (76\%), o que está de acordo com a literatura, assim como os sintomas predominantes foram também os mesmos descritos em outras publicações ${ }^{(2,11,25,34)}$. Os sintomas de impactação alimentar e disfagia, mais freqüentes em crianças maiores e adolescentes, observados na presente amostra, são os mais característicos e devem suscitar a hipótese diagnóstica de EE.

EE é definida pela presença de grande número de eosinófilos no epitélio escamoso esofágico, com sintomas esofágicos correspondentes $^{(2,12,25,34)}$. Acreditava-se que esta era uma entidade rara, mas progressivamente novos casos foram sendo relatados, principalmente em países desenvolvidos, que se acumularam na literatura médica ${ }^{(10,11,34,36,50)}$.

Estudos clínicos têm sugerido que os distúrbios eosinofílicos, especialmente a EE, estão aumentando em incidência ${ }^{(1,9,32,47)}$. Infiltração eosinofílica do esôfago é observada em pacientes com refluxo gastroesofágico, reações a drogas, infecções, gastroenterite e esofagite eosinofílicas ${ }^{(1,19,38)}$. Os eosinófilos são integrantes do sistema imune da mucosa gastrointestinal e as doenças eosinofílicas do tubo digestivo são distúrbios primariamente alérgicos, poligênicos, que envolvem mecanismos puramente mediados por IgE ou respostas retardadas, celulares ${ }^{(1,38)}$. Estudos têm demonstrado o papel contributivo das citocinas interleucina-5 (IL-5), eotaxinas, IL-4, IL-13 e STAT6, comprovando o papel da resposta imune do tipo Th2 na patogênese da $\mathrm{EE}^{(1,19,36,38,41)}$. GUPTA et al. ${ }^{(17)}$ sugerem que a EE é primariamente uma resposta Th2 seletiva de IL-5, com possível componente Th1 e papel importante de substâncias quimioatrativas de eosinófilos. A IL-5 tem importante função em regular a produção, a diferenciação, o recrutamento, a ativação e a sobrevida dos eosinófilos ${ }^{(1,14,19,27,41)}$. BLANCHARD et al. ${ }^{\left({ }^{8}\right)}$ demonstraram que a eotaxina- 3 tem papel crítico como molécula efetora na EE. Como já é bem conhecido que a EE e as síndromes hipereosinofílicas são freqüentemente associadas com uma expressão aumentada da IL-5, a neutralização da IL-5, através de anticorpos anti-IL-5 
(mepolizumab) tem sido demonstrada como terapia promissora no tratamento dessas entidades ${ }^{(42)}$.

Os distúrbios eosinofílicos gastrointestinais (EE, gastroenterite eosinofílica, colite eosinofílica) constituem um grupo de enfermidades caracterizadas por sintomas gastrointestinais e infiltrado eosinofílico mucoso significativo ${ }^{(15,16,39)}$. Embora a causa dessas doenças seja ainda desconhecida, muitos pacientes respondem ao manejo nutricional, implicando as alergias alimentares como fator associado importante ${ }^{(6,39)}$. A EE é uma entidade caracterizada por sintomas esofágicos, semelhantes aos da doença por refluxo gastroesofágico e importante eosinofilia esofágica, que não responde somente ao tratamento com bloqueadores da secreção ácida gástrica ${ }^{(13,16)}$. Esse infiltrado inflamatório eosinofílico não ocorre no estômago e no duodeno, nem em outras porções do trato gastrointestinal. Utiliza-se esse critério diagnóstico de sintomas e infiltrado eosinofílico esofágico, sem eosinofilia em mucosa antral e duodenal.

Normalmente, a mucosa esofágica não contém eosinófilos e a presença de mais do que 20 eosinófilos por campo de grande aumento é considerada como marcador da EE. Na esofagite de refluxo, o número habitual de eosinófilos é de aproximadamente 1 a, no máximo, 10 eosinófilos por campo de grande aumento ${ }^{(11,49)}$. Pacientes com contagens entre 10 e 20 são considerados, muitas vezes, com diagnóstico duvidoso, situação esta ainda controversa ${ }^{(11)}$. ESPOSITO et al. ${ }^{(1)}$ relataram média de um eosinófilo por campo de grande aumento, variando de 0 a 13, em 198 crianças com esofagite de refluxo. Nesse estudo, apenas $4,5 \%$ dos pacientes apresentavam mais do que cinco eosinófilos por campo. Já NGO et al. ${ }^{(30)}$ apresentaram três pacientes com mais de 20 eosinófilos por campo, que responderam ao tratamento com IBP, configurando esofagite de refluxo e não-eosinofílica. Esses autores ${ }^{(30)}$ sugerem que antes do diagnóstico de EE, seja feito tratamento com IBP, para excluir que os achados de eosinofilia na mucosa esofágica sejam decorrentes de esofagite péptica, aspecto ainda discutido na literatura atual. Provavelmente, a EE possa ser complicada ou potencializada pela presença do refluxo concomitante e vice-versa, já que o infiltrado eosinofílico causa dismotilidade esofágica ${ }^{(29)}$. De qualquer maneira, aceita-se atualmente que o diagnóstico seja estabelecido pelo número maior do que 20 de eosinófilos intraepiteliais por campo de grande aumento ${ }^{(18,49)}$.

Todos os pacientes desta casuística apresentavam mais de 20 eosinófilos por campo e esse foi considerado o critério diagnóstico. Não se pode considerar a não resposta ao IBP, pois vários pacientes receberam os dois tratamentos simultaneamente.

Existem estudos atuais que avaliam a utilidade de outros parâmetros para diferenciar EE de esofagite de refluxo ${ }^{(18)}$. Assim, RAVELLI et al. ${ }^{(37)}$ demonstraram que a dilatação dos espaços intercelulares é um achado maior em esofagites, independente da causa. Já STEINER et al. ${ }^{(44)}$, estudando uma coorte de pacientes pediátricos, encontraram que a hiperplasia das células da camada basal é mais acentuada na EE do que nas esofagites de refluxo. KIRSCH et al. ${ }^{(21)}$ sugerem que a contagem de mastócitos intraepiteliais e das células produtoras de IgE da mucosa esofágica podem diferenciar EE e refluxo e talvez definir um subgrupo de pacientes com doença do refluxo, no qual há componente alérgico.
Os pacientes que receberam curso de esteróides inalatórios deglutidos, apresentaram boa resposta clínica. Os que foram submetidos a endoscopias e biopsias de controle apresentaram diminuição significativa do número de eosinófilos (de uma média de 32 eosinófilos/campo para 4/campo). Se o estudo de NGO et al. ${ }^{(30)}$ vier a se confirmar, os critérios diagnósticos de EE deverão mudar, mas até o presente momento todos os autores consideram o critério histológico, com contagem do número de eosinófilos, como o mais importante.

Dietas de eliminação são muitas vezes inconvenientes e difíceis de serem cumpridas, principalmente devido a palatabilidade e custos, em pacientes cujos sintomas não são necessariamente incapacitantes. As dietas elementares podem, muitas vezes, comprometer mais a qualidade de vida desses pacientes do que os sintomas da própria doença. Além disso, os pacientes que são submetidos a dieta de eliminação, apresentam recurrência dos sintomas quando voltam à dieta polimérica, como aconteceu com um dos pacientes desta série. As dietas de restrição podem ser uma alternativa, desde que haja identificação de alimentos envolvidos em reações alérgicas, por testes cutâneos.

Existem estudos indicando que alérgenos respiratórios e de pele poderiam contribuir como causa das EE, pois pacientes com esta enfermidade apresentam doenças alérgicas respiratórias $\operatorname{associadas}^{(1,33,43)}$. Nesses casos, seria difícil excluir o alérgeno em questão.

Corticóides orais apresentam vários efeitos colaterais e complicações. O inibidor de leucotrienos - montelukast parece ter efeito protetor, mas a recidiva é grande após sua retirada. O tratamento com esteróides tópicos parece ser a alternativa mais segura e eficaz, ainda que a técnica de aplicação possa gerar problemas ${ }^{(7,24,48)}$.

Não há critérios objetivos para avaliar a resposta ao tratamento, os estudos têm empregado acompanhamento clínico, subjetivo e endoscópico/histológico para essa avaliação ${ }^{(50)}$. Consideram-se os sintomas clínicos e a contagem de eosinófilos pós-tratamento como marcadores de melhora.

KONIKOFF et al. ${ }^{(22)}$ conduziram o primeiro estudo multicêntrico, prospectivo e controlado por placebo, para determinar a eficácia do propionato de fluticasona no tratamento da EE. Trinta e seis pacientes foram selecionados para receber $880 \mu \mathrm{g}$ de fluticasona spray deglutido (21 pacientes) ou placebo (15 pacientes). $\mathrm{O}$ desfecho do estudo foi remissão histológica, com um ou menos eosinófilo/campo em todos os campos estudados. Cinqüenta por cento do grupo tratamento atingiu remissão histológica, comparado com apenas $9 \%$ do grupo placebo. Eles notaram que o tratamento com fluticasona diminuiu o número de linfócitos T CD8+ e dos mastócitos no esôfago proximal e distal. Esse resultado foi mais proeminente nos pacientes não-alérgicos e mais jovens. Esses autores concluíram que o propionato de fluticasona spray deglutido é eficaz em induzir remissão histológica em $\mathrm{EE}^{(22)}$.

Não há relatos na literatura de risco de malignização ou evolução para doença eosinofílica mais extensa ${ }^{(50)}$. $\mathrm{Na}$ população adulta, a doença tende a ser estável, sem efeitos significativos na morbidade e na mortalidade desses pacientes ${ }^{(3,}$ 35, 45). STRAUMANN et al. ${ }^{(45)}$ relataram a história natural de 30 pacientes adultos, acometidos de EE, com média de idade 
de 41 anos. Todos evoluíram clinicamente bem, sem perdas importantes de peso. Como 29 pacientes haviam se apresentado com disfagia, 11 necessitaram novas dilatações, dos quais 10 tiveram diminuição ou cura da mesma. Em termos de qualidade de vida, apenas 1 paciente relatou impacto negativo na sua vida profissional, 14 não apresentaram impacto significativo e 15 relataram influência menor da doença.

ESPOSITO et al. ${ }^{(11)}$ acompanharam, por 4 anos, sete crianças com EE, com idades entre 6 meses e 14 anos, todos tratados com fluticasona: dois apresentaram recidivas em 1 ano e 4 anos póstratamento, que melhorou com um segundo curso de medicação. Todos apresentaram crescimento normal. Dois pacientes não seguiram o tratamento e por isso tiveram resposta endoscópica e histológica inadequada.

Os pacientes desta casuística ainda apresentam pouco tempo de evolução de doença. Todos eles, entretanto, apresentaram resposta clínica favorável ao tratamento inicial com esteróides inalatórios deglutidos.

ORENSTEIN et al. ${ }^{(34)}$ demonstraram que um terço dos pacientes ficaram assintomáticos sem tratamento, enquanto LIACOURAS et al. ${ }^{(24)}$ encontraram $50 \%$ de recidiva após 1 ano de tratamento com corticosteróide oral.
Devido à história natural aparentemente benigna dessa entidade, na qual muitos pacientes parecem não sofrer impacto significativo na qualidade de vida, as terapias necessitam ser validadas em ensaios clínicos, com análise de custo-benefício.

STRAUMANN e SIMON ${ }^{(4)}$ apresentaram dados, em adultos, que mostraram real aumento dessa enfermidade crônica e inflamatória do esôfago e salientaram que ela pode, rapidamente, tornar-se tão freqüente quanto a doença inflamatória intestinal, pelo menos nos países desenvolvidos. Não há dúvidas de que essa doença está adquirindo papel muito importante em todo o mundo ${ }^{(40)}$.

A patogênese, as causas, a história natural dessa doença e a resposta ao tratamento em longo prazo ainda necessitam esclarecimentos, mas a $\mathrm{EE}$, que pode não ter sido valorizada no passado, certamente impõe-se como entidade própria, constituindo-se atualmente em uma das mais prevalentes doenças esofagianas.

O presente estudo deve servir de motivação aos pediatras, para suspeitarem do diagnóstico de EE e discutirem as modalidades de diagnóstico e terapêuticas, aos endoscopistas, para coletarem biopsias de esôfago, mesmo na ausência de anormalidades macroscópicas, e aos patologistas, para analisarem e quantificarem a infiltração eosinofílica do esôfago.

Ferreira CT, Vieira MC, Vieira SMG, Silva GS, Yamamoto DR, Silveira TR. Eosinophilic esophagitis in 29 pediatric patients. Arq Gastroenterol. 2008;45(2):141-6.

ABSTRACT - Background - Eosinophilic esophagitis is a recently described entity with esophageal symptoms like gastroesophageal reflux disease and significant esophageal eosinophilic infiltration. Aim - To present our clinical series of 29 children with eosinophilic esophagitis, describing the clinical and diagnostic features, treatment and outcome. Methods - We describe 29 patients (22 boys), 1-18 years-old, with 20 eosinophils per high-power field in esophageal biopsy specimens and absence of eosinophilic inflammation in the stomach and duodenum. Evaluation of the clinical, endoscopic and histologic findings, treatment and outcome was undertaken. Results - The most common presenting symptoms included vomiting in 15 patients (52\%) and abdominal pain in 11 patients (38\%). Children under the age of 4 years presented with feeding disorder and failure to thrive. Patients between 5 and 8 years of age presented commonly with abdominal pain or symptoms that may be associated with reflux (heartburn and/or vomiting). Patients over the age of 8 presented most often with abdominal pain, dysphagia and occasional food impaction. Endoscopic features included vertical furrowing in 14 patients (48\%), whitish papules in 12 (41\%), corrugated rings in 2 patients (7\%) and esophageal erosions in 3 patients (10\%). In seven patients endoscopy was normal (24\%). Treatment included swallowed fluticasone propionate in 19 patients and restriction diet in 7 patients. Patients who returned for follow-up had either improvement or remission of symptoms. After treatment, endoscopic biopsies were repeated in 11 patients, and a significant decrease in esophageal eosinophil counts was observed. Conclusions - The diagnosis of eosinophilic esophagitis must be considered when symptoms of reflux do not respond to conventional treatment. Upper gastrointestinal endoscopy must be complemented by a detailed analysis of histologic findings and eosinophil counts.

HEADINGS - Esophagitis. Eosinophilia. Child. 


\section{REFERÊNCIAS}

1. Akei HS, Mishra A, Blanchard C, Rothenberg ME. Epicutaneous antigen exposure primes for experimental eosinophilic esophagitis in mice. Gastroenterology. 2005;129:985-94.

2. Antonioli DA, Furuta GT. Allergic eosinophilic esophagitis: a primer for pathologists Semin Diagn Pathol. 2005;22:266-72.

3. Arora AS, Perrault J, Smyrk TC. Topical corticosteroid treatment of dysphagia due to eosinophilic esophagitis in adults. Mayo Clin Proc. 2003;78:830-5.

4. Assa'ad AH, Putnam PE, Collins MH, Akers RM, Jameson SC, Kirby CL, Buckmeie BK, Bullock JZ, Collier AR, Konikoff MR, Noel RJ, Guajardo JR, Rothenberg ME. Pediatric patients with eosinophilic esophagitis: a 8-year follow-up. J Allergy Clin Immunol. 2007;119:731-8.

5. Attwood SE, Smyrk TC, Demeester TR, Jones JB. Esophageal eosinophilia with dysphagia. A distinct clinicopathologic syndrome. Dig Dis Sci. 1993;38:109-16.

6. Bischoff S, Crowe SE. Gastrointestinal food allergy: new insights into pathophysiology and clinical perspectives. Gastroenterology. 2005;128:1089-113.

7. Blanchard C, Wang N, Rothenberg ME. Eosinophilic esophagitis: pathogenesis, genetics, and therapy. J Allergy Clin Immunol. 2006;118:1054-9.

8. Blanchard C, Wang N, Stringer KF, Mishra A, Fulkerson PC, Abonia JP, Jameson SC, Kirby C, Konikoff MR, Collins MH, Cohen MB, Akers R, Hogan SP, Assa'ad AH, Putnam PE, Aronow BJ, Rothenberg ME. Eotaxin-3 and a uniquely conserved geneexpression profile in eosinophilic esophagitis. J Clin Invest. 2006;116:536-47.

9. Cherian S, Smith NM, Forbes DA. Rapidly increasing prevalence of eosinophilic oesophagitis in Western Australia. Arch Dis Child. 2006;91:1000-4.

10. Desai TK, Stecevic V, Chang CH, Goldstein NS, Badizadegan K, Furuta GT. Association of eosinophilic inflammation with esophageal food impaction in adults. Gastrointest Endosc. 2005;61:795-801.

11. Esposito S, Marinello D, Paracchini R, Guidali P, Oderda G. Long-term follow-up of symptoms and peripheral eosinophil counts in seven children with eosinophilic esophagitis. J Pediatr Gastroenterol Nutr. 2004;38:452-6.

12. Fox VL, Nurko S, Furuta GT. Eosinophilic esophagitis: it's not just kid's stuff. Gastrointest Endosc. 2002;56:260-70.

13. Furtado GB, Herculano Jr. JRL. Esofagite eosinofilica: aspectos clínicos, endoscópicos e terapêuticos em 10 casos. GED Gastrointest Endosc Dig. 2004;23:249-54.

14. Furuta GT. Emerging questions regarding eosinophil's role in the esophago-gastrointestinal tract. Curr Opinion Gastroenterol. 2006;22:658-63.

15. Furuta GT. Eructations from eosinophils. Gastroenterology. 2006;131:1629-31.

16. Furuta GT, Straumann A. Review article: the pathogenesis and management of eosinophilic oesophagitis. Aliment Pharmacol Ther. 2006;24:173-82.

17. Gupta SK, Fitzgerald JF, Kondratyuk T, HogenEsch H. Cytokine expression in normal and inflamed esophageal mucosa: a study into the pathogenesis of allergic eosinophilic esophagitis. J Pediatr Gastroenterol Nutr. 2006;42:22-6.

18. Hassall E. Esophageal biopsy in children--essential, valuable, or a waste of time? It all depends. J Pediatr Gastroenterol Nutr. 2005;41(Suppl 1):s24-s7.

19. Hogan SP, Rothenberg ME. Review article. The eosinophil as a therapeutic target in gastrointestinal disease. Aliment Pharmacol Ther. 2004;20:1231-40.

20. Kelly KJ, Lazenby AJ, Rowe PC, Yardley JH, Perman JA, Sampson HA. Eosinophilic esophagitis attributed to gastro-oesophageal reflux: improvement with an amino acidbased formula. Gastroenterology. 1995;109:1503-12.

21. Kirsch R, Bokhary R, Marcon MA, Cutz E. Activated mucosal mast cells differentiate eosinophilic (allergic) esophagitis from gastroesophageal reflux disease. J Pediatr Gastroenterol Nutr. 2007;44:20-6.

22. Konikoff MR, Noel RJ, Blanchard C, Kirby C, Jameson SC, Buckmeier BK, Akers R, Cohen MB, Collins MH, Assa'ad AH, Aceves SS, Putnam PE, Rothenberg ME. A randomized, double-blind, placebo-controlled trial of fluticasone propionate for pediatric eosinophilic esophagitis. Gastroenterology. 2006;131:1381-91.

23. Landres RT, Kuster GG, Strum WB. Eosinophilic esophagitis in a patient with vigorous achalasia. Gastroenterology. 1978;74:1298-301.

24. Liacouras CA, Wenner WJ, Brown K, Ruchelli E. Primary eosinophilic esophagitis in children: successful treatment with oral corticosteroids. J Pediatr Gastroenterol Nutr. 1998;26:380-5.

25. Liacouras CA, Spergel JM, Ruchelli E, Verma R, Mascarenhas M, Semeao E, Flick J, Kelly J, Brown-Whitehorn T, Mamula P, Markowitz JE. Eosinophilic esophagitis: a 10 year-experience in 381 children. J Clin Gastroenterol Hepatol. 2005;3:1198-206.

26. Liacouras CA. Eosinophilic esophagitis: treatment in 2005. Curr Opin Gastroentrol 2006;22:147-52.
27. Mishra A, Rothenberg ME. Intratracheal IL-13 induces eosinophilic esophagitis by an IL-5, eotaxin-1 and STAT6-dependent mechanism. Gastroenterology. 2003; 125:1419-27.

28. Mueller S, Aigner T, Neureiter D, Stolte M. Eosinophil infiltration and degranulation in oesophageal mucosa from adult patients with oesinophilic oesophagitis: a retrospective and comparative study on pathological biopsy. J Clin Pathol. 2006;59:1175-80.

29. Murch S. Allergy and dysmotility - causal or coincidental links? J Pediatr Gastroenterol Nutr. 2005;41:s14-s6.

30. Ngo P, Furuta GT, Antonioli DA, Fox VL. Eosinophils in the esophagus - peptic or allergic eosinophilic esophagitis? Case series of three patients with esophageal eosinophilia. Am J Gastroenterol. 2006;101:1666-70.

31. Nielsen RG, Fenger C, Bindslev-Jensen C, Husby S. Eosinophilia in the upper gastrointestinal tract is not a characteristic feature in cow's milk sensitive gastrooesophageal reflux disease. Measurement by two methodologies. J Clin Pathol. 2006;59:89-94.

32. Noel RJ, Putnam PE, Rothenberg ME. Eosinophilic esophagitis. N Engl J Med. 2004;351:940-1.

33. Onbasi K, Sin AZ, Doganavsargil B, Onder GF, Bor S, Sebik F. Eosinophil infiltration of the esophageal mucosa in patients with pollen allergy during the season. Clin Exp Allergy. 2005;35:1423-31.

34. Orenstein SR, Shalaby TM, Di Lorenzo C, Putnam PE, Sigurdsson L, Mousa H, Kocoshis SA. The spectrum of pediatric eosinophilic esophagitis beyond infancy: a clinical series of 30 children. Am J Gastroenterol. 2000;95:1422-30.

35. Parfitt JR, Gregor JC, Suskin NG, Jawa HA, Driman DK. Eosinophilic esophagitis in adults: distinguishing features from gastroesophageal reflux disease: a study of 41 patients. Mod Pathol. 2006;19:90-6.

36. Potter JW, Saeian K, Staff D, Massey BT, Komorowski RA, Shaker R, Hogan WJ Eosinophilic esophagitis in adults: an emerging problem with unique esophageal features. Gastrointest Endosc. 2004;59:355-61.

37. Ravelli AM, Villanacci V, Ruzzenenti N, Grigolato P, Tobanelli P, Klersy C, Rindi G Dilated intercellular spaces: a major morphological feature of esophagitis. J Pediatr Gastroenterol Nutr. 2006;42:510-5.

38. Rothenberg ME, Mishra A, Brandt EB, Hogan SP. Gastrointestinal eosinophils. Immunol Rev. 2001;179:139-55

39. Rothenberg ME. Eosinophilic gastrointestinal disorders (EGID). J Allergy Clin Immunol. 2004;113:11-28

40. Segundo GRS. Esophageal achalasia and eosinophilic esophagitis. J Pediatr. 2005:81:185-6

41. Shek LPC, Castro BR, Sampson HA, Beyer K. Humoral and cellular responses to cow milk proteins in patients with milk-induced IgE-mediated and non-IgE-mediated disorders. Allergy. 2005;60:912-9.

42. Simon D, Braathen LR, Simon HU. Anti-interleukin-5 antibody therapy in eosinophilic diseases. Pathobiology. 2005;72:287-92.

43. Simon D, Marti H, Heer P, Simon HU, Braathen LR, Straumann A. Eosinophilic esophagitis is frequently associated with IgE-mediated allergic airway diseases. J Allergy Clin Immunol. 2005;115:1090-2.

44. Steiner SJ, Kernek KM, Fitzgerald JF. Severity of basal cell hyperplasia differs in reflux versus eosinophilic esophagitis. J Pediatr Gastroenterol Nutr. 2006;42:506-9.

45. Straumann A, Spichtin HP, Grize L, Bucher KA, Beglinger C, Simon HU, Natural history of primary eosinophilic esophagitis: a follow-up of 30 adult patients for up to 11,5 years. Gastroenterology. 2003;25:1660-9.

46. Straumann A, Simon HU. Eosinophilic esophagitis: escalating epidemiology? J Allergy Clin Immunol. 2005;115:418.

47. Straumann A, Beglinger C. Eosinophilic esophagitis: the endoscopist's enigma. Gastrointest Endosc. 2006;63:13-5.

48. Teitelbaum JE, Fox VL, Twarog FJ, Nurko S, Antonioli D, Gleich G, Badizadegan K, Furuta GT. Eosinophilic esophagitis in children: immunopathological analysis and response to fluticasone propionate. Gastroenterology. 2002;122:1216-25.

49. Winter HA, Madara JL, Stafford RJ. Intraepithelial eosinophils: a new diagnostic criterion for reflux esophagitis. Gastroenterology. 1982;83:818-23.

50. Yan BM, Shafer EA. Eosinophilic esophagitis: a newly established cause of dysphagia. World J Gastroenterol. 2006;15:2328-34. 